\title{
Three Dimensional Position Detection Using a Two Dimensional PSD and a Marker Composed of Two Light Sources
}

\author{
Kosuke Onishi, Daisuke Tanaka, Shiyuan Yang \\ Kyushu Institute of Technology
}

\begin{abstract}
In recent years, the three-dimensional (3D) measurement is used in various fields. However, the conventional 3D measurement method has various problems such as complicated pre-set, slow processing and high price. In this study, in order to achieve the 3D measurement with simple preset, high speed processing and low price, we propose the 3D measurement method based on the new principle. It is the 3D position detection using a two-dimensional (2D) Position Sensitive Device (PSD) and a marker composed of two light sources. The major characteristics of the proposed method are as follows:

- Simple preset

- High speed detection

- Low price

- Not influenced by the reflectance of the object

We can convert from the 2D coordinate of the spot lights on the photosensitive area to the 3D coordinate of the marker by the proposed conversion formula. Moreover, the 2D PSD can receive the lights at the same time by using the light sources which is modulated frequency.

We performed experiments of four types to verify the measurement accuracy. We compared the experimental result of accuracy standard with the experimental results in the case of setting wide interval of light sources, using the high luminance light sources and controlling the photographing magnification.

From four experiments, we show that the proposed method is an effective 3D measurement method and measurement accuracy is largely improved by three proposed solutions
\end{abstract}

Keywords: 3D measurement, 2D PSD, marker.

\section{Introduction}

The 3D measurement method have various applications such as games, CG creation, structure research, cultural heritage and ruins investigation, robot vision and medical equipment in recent years. In the future, the demand for the 3D measurement would increase with the development of the CG technology. The 3D measurement method can be largely divided into two types, the passive and active measurement.

\subsection{Passive measurement method}

The passive measurement uses the camera only. The passive measurement is divided into the depth from focus method and the multi camera stereo method. These non-contact measurement techniques have found widespread use in heritage, environment modeling, virtual reality, and many industrial fields ${ }^{(1)}$. The depth from focus method uses the focal length that varies depending on the object positions, and the distance between the object and the camera is calculated based on the focal length. The multi camera stereo method uses multiple cameras, and the 3D position of the object is calculated based on the principle of triangulation. Because the depth from focus method is necessary to change the focal length by the sequential lens driving, measurement for the moving object and real time measurement are difficult. In the multi camera stereo method, preset is important because the calibration to determine the positional relationship of multiple cameras affects the measurement accuracy. However, it is complicated. Also, the multi camera stereo method takes a long calculation time because the image processing is complicated. Furthermore, the price of the multi camera stereo method is than other methods because it uses multiple cameras.

\subsection{Active measurement method}

On the other hand, the active measurement uses the light, radio wave or sound wave and the camera. The active measurement is divided into the light radar method and the 
active stereo method. The light radar method uses the time difference between projecting the light and receiving the light. The active stereo method uses the positional relationship of the spot light on the object, the camera and the light source. The active stereo method and the light radar method are not able to measure the $3 \mathrm{D}$ position when the object has an optical transmission or light absorption because these methods use the light reflection on the object surface. The active stereo method can determine the relative displacement of the object position. However, the active stereo method can't determine absolute distance. Also, the active stereo method takes a long calculation time because this method uses the changed pattern of the irradiated light on the object surface.

\subsection{Purpose of the research}

In order to improve disadvantages of conventional methods and achieve the 3D measurement with simple preset, high speed detection, low price and not influenced by the reflectance of the object, we propose the $3 \mathrm{D}$ measurement method based on the new principle. It is the 3D position detection using a 2D PSD and a marker composed of two light sources.

\section{Principle}

First, we project the light by the marker toward the photosensitive area. We call this part the light projecting unit. Second, projected light is imaged by an optical lens on the photosensitive area. We call this part the light receiving unit. Third, the four output signals from the 2D PSD are $\mathrm{A} / \mathrm{D}$ converted, and these data are calculated by the PC. Then, the 2D coordinate of the spot light on the photosensitive area is detected. The $3 \mathrm{D}$ coordinate of the marker is calculated based on the $2 \mathrm{D}$ coordinate data. We call this part the calculation unit.

We show positional relationship between the marker and a 2D PSD in Fig. 1. Fig. 1(a) is schematic view of the measurement system, Fig. 1 (b) is the $X-Z$ plane view of the measurement system, and Fig. 1 (c) is the $Y-Z$ plane view of the measurement system.

\subsection{Asynchronous measurement method}

If the 2D PSD receives the light of sources at the same time, four output signals from a 2D PSD are the synthesized wave of different frequency sine waves. Four spectrums are obtained in the each modulation frequency of light sources by Fast Fourier Transform (FFT). Then, the Fourier

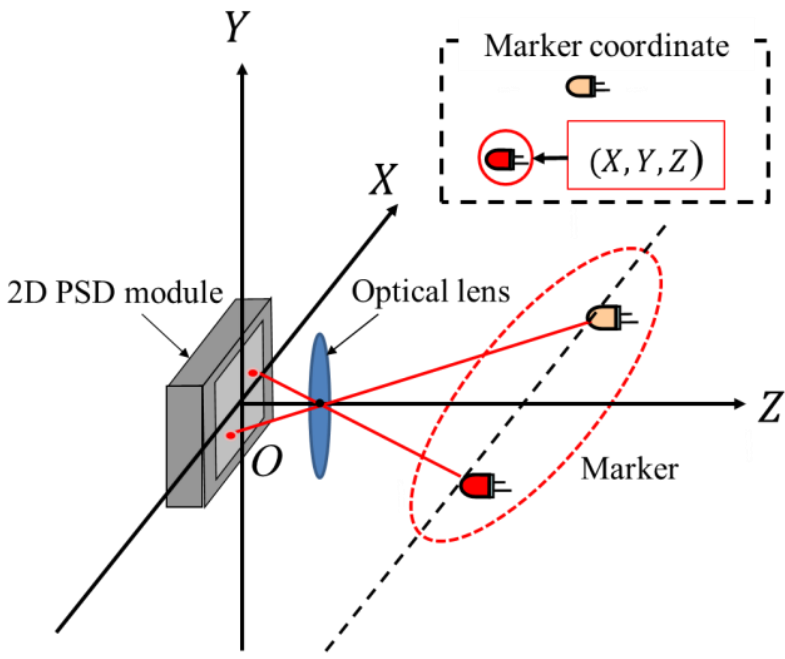

(a) Schematic view

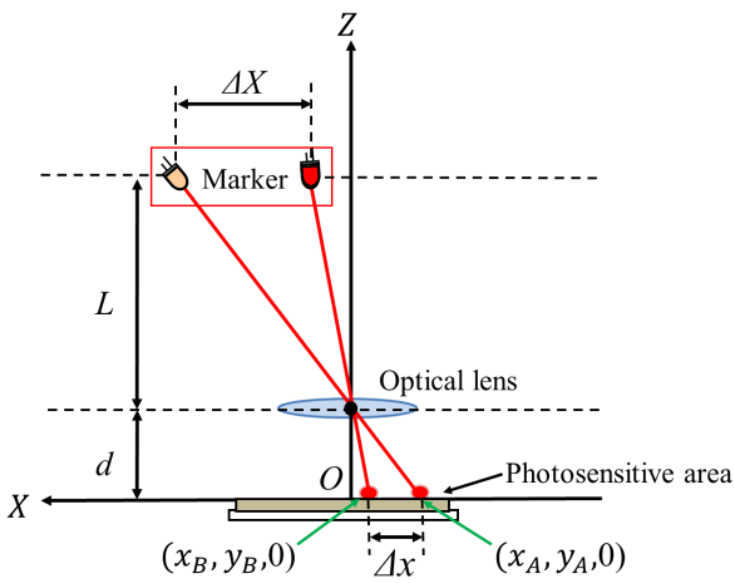

(b) X-Z plane

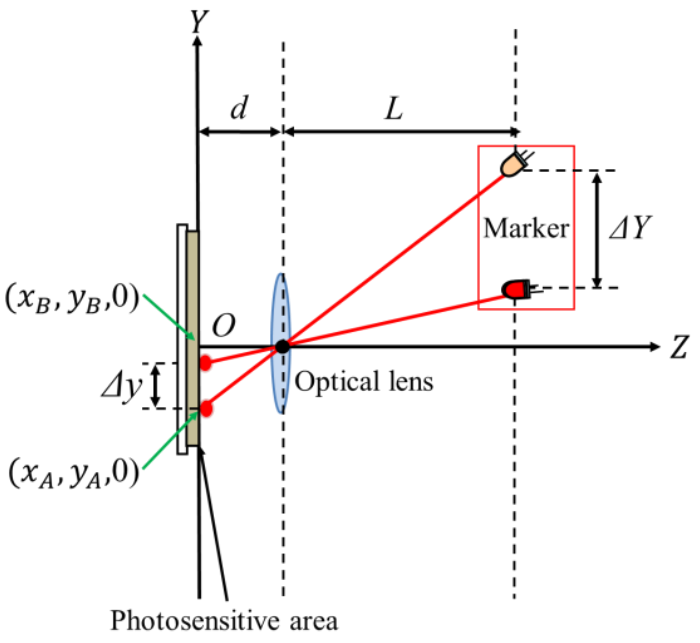

(c) Y-Z plane

Fig. 1. Positional relationship between the marker and the 2D PSD. 
amplitude spectrum is converted to the $2 \mathrm{D}$ coordinate $(x, y)$ on the photosensitive area by using conversion formula. Four spectral peaks are $X 1, X 2, Y 1, Y 2$. The photosensitive area is a square $c[\mathrm{~mm}]$ on a side.

$$
\begin{aligned}
& x=\frac{(X 2+Y 1)-(X 1+Y 2)}{X 1+X 2+Y 1+Y 2} \times \frac{c}{2} \\
& y=\frac{(X 2+Y 2)-(X 1+Y 1)}{X 1+X 2+Y 1+Y 2} \times \frac{c}{2}
\end{aligned}
$$

Therefore, even if the 2D PSD receives the light of sources at the same time, this method can detect the $2 \mathrm{D}$ coordinate.

\subsection{D measurement method}

From Fig. 1 (b) and (c), the $\Delta x[\mathrm{~mm}]$ is the interval of two spot lights by the marker on the photosensitive area. Also, the $\Delta X[\mathrm{~mm}]$ is the arbitrary interval of two light sources of a marker. Furthermore, the distance $L[\mathrm{~mm}]$ is calculated based on the similarity relationship of triangle as follows:

$$
L=\frac{\Delta X}{\Delta x} d
$$

The $X$ coordinate and the $Y$ coordinate are calculated based on the similarity relationship of triangle as follows:

$$
\begin{aligned}
& X=\frac{L}{d} x_{B} \\
& Y=\frac{L}{d} y_{B} \\
& Z=L+d
\end{aligned}
$$

However, this principle has the condition. The marker must be arranged in parallel to the photosensitive area.

\subsection{Preset of the distance $d$}

The 2D coordinate $\left(x_{B}, y_{B}\right)$ of spot light on the photosensitive area, the interval $\Delta x$, the distance $L$, the $3 \mathrm{D}$ coordinate of the light source $(X, Y, Z)$ are successively calculated during the system operation. However, the light source interval $\Delta X$ and the distance $d$ are required to be preset. The light source interval $\Delta X$ is arbitrary value. The distance $d$ was experimentally calculated because it is not any value. From the equation (3) and (4), the equation for calculating the distance $d$ is as follows.

$$
d=\left(1+\frac{\Delta x}{\Delta X}\right)^{-1} Z
$$

In this measurement system, the $Z$ coordinate of the light source was measured by using the ruler, the light source interval $\Delta X$ was set $5[\mathrm{~cm}]$. The spot light interval on the photosensitive area is $\Delta x$. We set the $X$ coordinate of the marker to each $-2.5[\mathrm{~cm}]$ and $2.5[\mathrm{~cm}]$, the $Y$ coordinate to 0

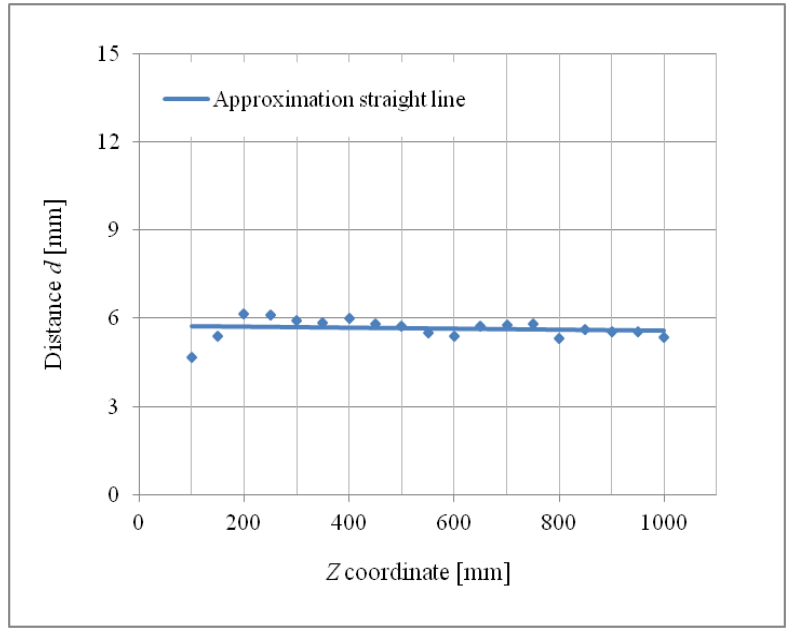

Fig. 2. Measurement result of the distance $d$.

[cm]. And we changed the $Z$ coordinate from $10[\mathrm{~cm}]$ to $100[\mathrm{~cm}]$ at an interval of $5[\mathrm{~cm}]$. We show measurement result of the distance $d$ in Fig. 2.

From Fig. 2, we found that the distance $d$ was the certain value. Therefore, the distance $d$ was set 5.70 [mm] based on the average value of the measured values.

\section{Measurement system}

The measurement system is composed of the light projecting unit, the light receiving unit and the calculating unit. We show the measurement system configuration diagram in Fig. 3.

\subsection{Light projecting unit}

The frequency modulation circuit can modulate the frequency of the light source into an optional frequency. And, we project the light that frequency is modulated toward the light receiving unit.
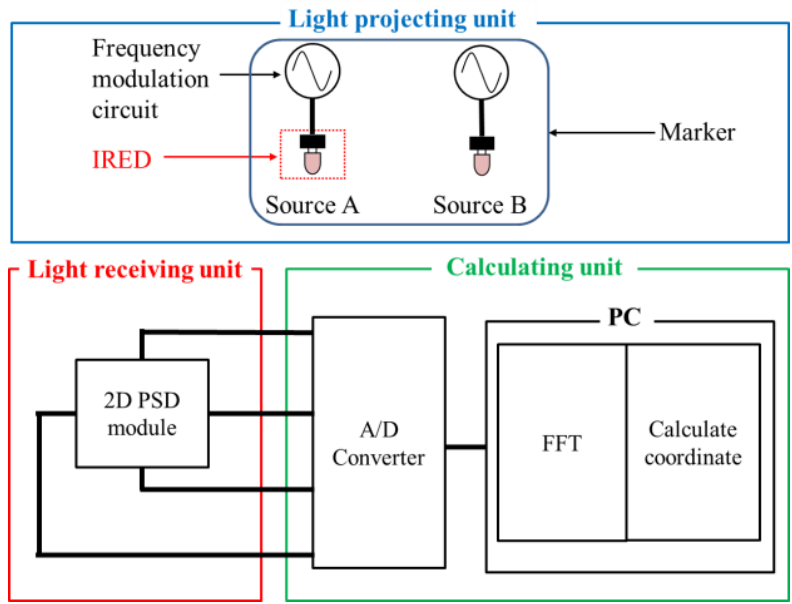

Fig. 3. Measuring system configuration diagram 


\subsection{Light receiving unit}

The projected light passes through the optical lens, and is imaged on the photosensitive area of the light receiving unit. Then, electric current corresponding to the spotlight coordinate on the photosensitive area is output from the 2D PSD. The electric current is converted voltage in the 2D module. Four signals output from the 2D PSD module are sent to the $A / D$ converter of the calculating unit to detect the 2D coordinate of the spot light on the photosensitive area.

\subsection{Calculating unit}

The amplitude data converted by the A/D converter is stored in the memory of the A/D converter. Then, 1024 amplitude data are taken from each of the output signal of the 2D PSD module, these data are sent to the PC after 4096 amplitude data are stored in memory.

The FFT calculation is executed targeting the amplitude data on the PC. Then, 4 spectrums are obtained at the modulation frequency of each light source from the composite wave. 3D coordinate $(X, Y, Z)$ of each light source is determined by using their spectral peak voltages.

\section{Experiments}

We verified accuracy of the 3D coordinate detection. We performed experiments of four types. Theoretical value of the light source coordinate is the value that was measured using the graph paper and the ruler. We measured under fluorescent lamps. The marker was used the light source modulated to $10[\mathrm{kHz}]$ and $9[\mathrm{kHz}]$. Because the $Y$ coordinate are detected by the same principle as principle of the $X$ coordinate, we omit the $Y$ coordinate measurement.

\subsection{Accuracy verification of the normal type}

This experiment was the normal type. The result of this experiment was accuracy standard. We verified accuracy of the 3D coordinate detection. The radiant intensity of infrared-emitting diodes (IREDs) was $22[\mathrm{~mW} / \mathrm{Sr}]$. The light source interval $\Delta X$ of a marker was $5[\mathrm{~cm}]$.

First, we verified measurement accuracy of the $Z$ coordinate of the marker. The $X$ coordinate of two light sources of a marker were set $-2.5[\mathrm{~cm}]$ and $+2.5[\mathrm{~cm}]$, The $Y$ coordinate was set $0[\mathrm{~cm}]$. Moreover, the $Z$ coordinate was changed from $10[\mathrm{~cm}]$ to $100[\mathrm{~cm}]$ at an interval of 5 [cm]. We show the $Z$ coordinate measurement result in Fig. 4.
Second, we verified measurement accuracy of the $X$ coordinate of the marker. The $Y$ coordinate of the marker were set $0[\mathrm{~cm}]$, the $Z$ coordinate was $20[\mathrm{~cm}]$. Moreover, the $X$ coordinate moved from $-20[\mathrm{~cm}]$ to $+20[\mathrm{~cm}]$ at an interval of $5[\mathrm{~cm}]$. In the similar way, the $X$ coordinate was measured when the $Z$ coordinate was $50[\mathrm{~cm}]$ and $80[\mathrm{~cm}]$. We show the $X$ coordinate measurement result when the $Z$ coordinate was $20[\mathrm{~cm}], 50[\mathrm{~cm}], 80[\mathrm{~cm}]$ in Fig. 5(a), (b), (c).

We describe the measurement result in the following. First, we describe the $Z$ coordinate measurement result. The maximum error of the $Z$-coordinate measurement results was $133[\mathrm{~mm}]$, the maximum error rate was 17 [\%].

Second, we describe the $X$ coordinate measurement result when the $Z$ coordinate was $20[\mathrm{~cm}]$. 3D coordinate could not be measured because the marker were not entered into the view angle of the camera when the $X$ coordinate was $15[\mathrm{~cm}]$ or more in absolute value. Furthermore, the maximum measurement error of the maximum measurement error rate was 17.7 [\%] within the measuring range.

Third, when the $Z$ coordinate was $50[\mathrm{~cm}]$, the maximum measurement error of the $X$-coordinate measurement results was $63.3[\mathrm{~mm}]$, the maximum measurement error rate was 31.7 [\%].

Finally, when the $Z$ coordinate was $80[\mathrm{~cm}]$, the maximum measurement error of the $X$-coordinate measurement results was $45.9[\mathrm{~mm}]$, the maximum measurement error rate was 23.0 [\%].

From the above, the measurement error markedly appeared as the light sources separated farther from the photosensitive area. Therefore, the measurement error cause was correlation with the incident light quantity.

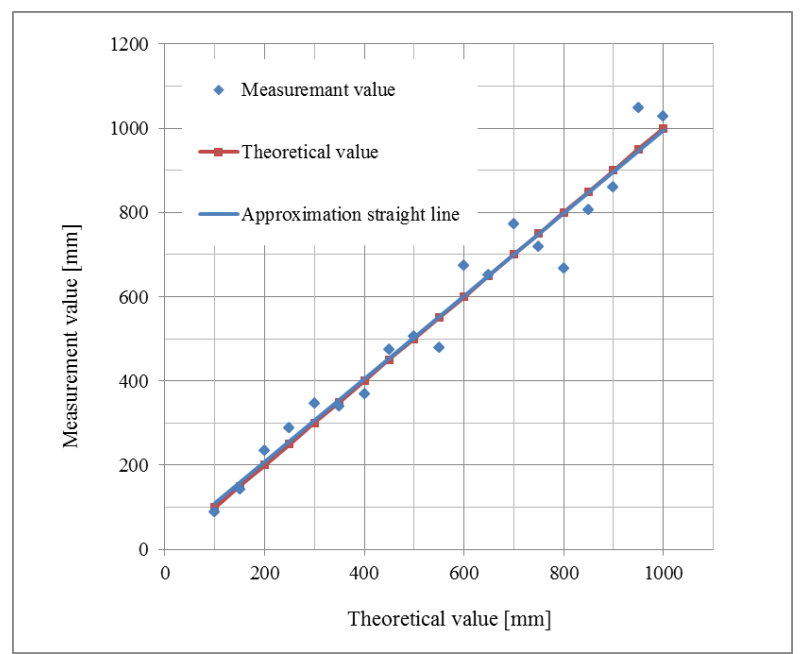

Fig. 4. Measurement result of the $Z$ coordinate. 


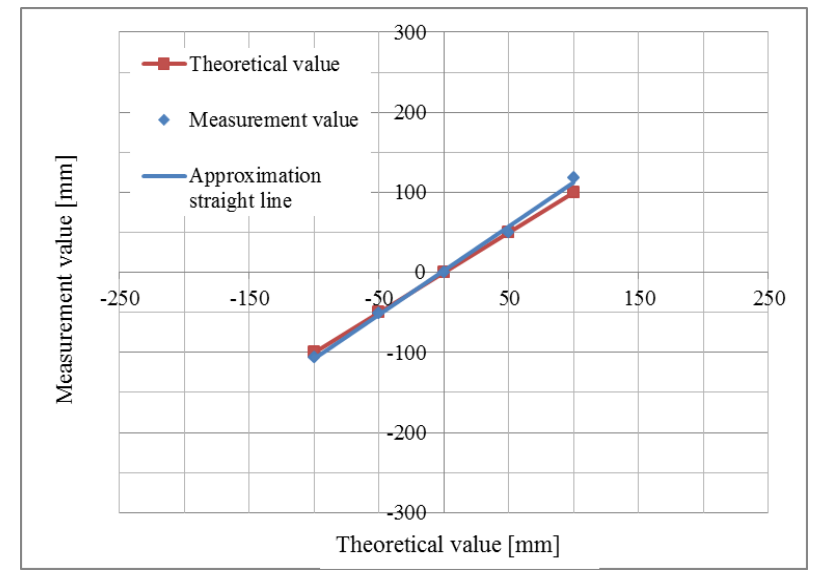

(a) $\mathrm{Z}=20[\mathrm{~cm}]$

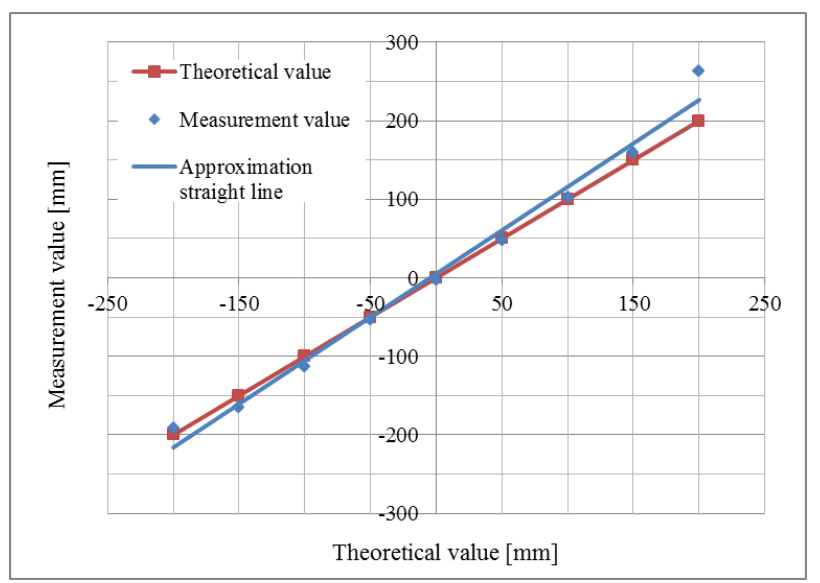

(b) $\mathrm{Z}=50[\mathrm{~cm}]$

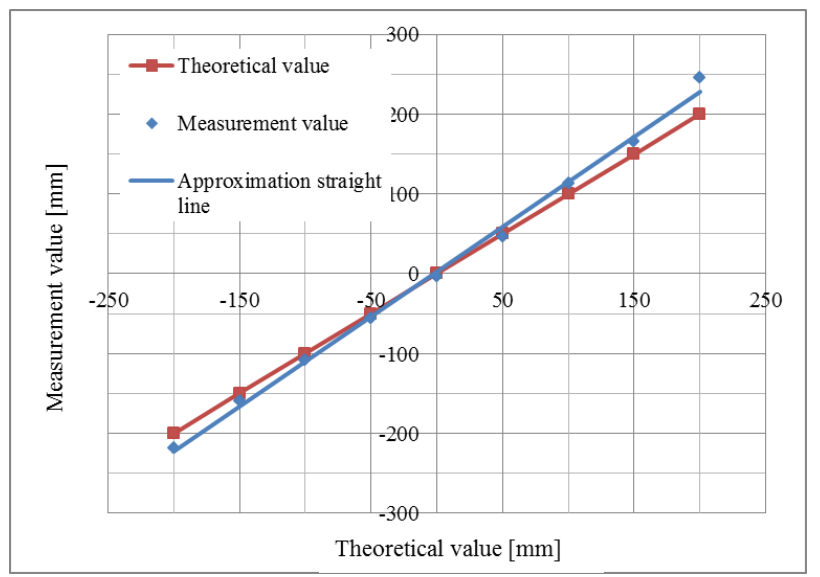

(c) $\mathrm{Z}=80[\mathrm{~cm}]$

Fig. 5. Measurement result of the $X$ coordinate.

Furthermore, another measurement error cause is that the spot light size was larger than photosensitive area. The graph of the average value almost coincided with the approximation graph of the theoretical value. Therefore, the proposed method is an effective 3D measurement method.

\subsection{Accuracy verification of the wide type}

This experiment was the wide type. This type was the experiment in the case of setting wide interval $\Delta X$ of light sources. We compared the measurement accuracy of the wide type and the normal type. In this experiment, the light source interval $\Delta X$ was $10[\mathrm{~cm}]$ or $20[\mathrm{~cm}]$. Also, the radiant intensity of IREDs was 22 [mW/Sr].

First, we measured the $Z$ coordinate of the marker when the light source interval $\Delta X$ was 10 [cm]. The $X$ coordinate of two light sources of a marker were $-5[\mathrm{~cm}]$ and $+5[\mathrm{~cm}]$, The $Y$ coordinate was $0[\mathrm{~cm}]$. Moreover, the $Z$ coordinate was moved from $10[\mathrm{~cm}]$ to $100[\mathrm{~cm}]$ at an interval of $5[\mathrm{~cm}]$.

Second, we measured the $Z$ coordinate of the marker when the light source interval $\Delta X$ was $10[\mathrm{~cm}]$. The $X$ coordinate of two light sources of a marker were -10 [cm] and $+10[\mathrm{~cm}]$, The $Y$ coordinate was $0[\mathrm{~cm}]$. Moreover, the

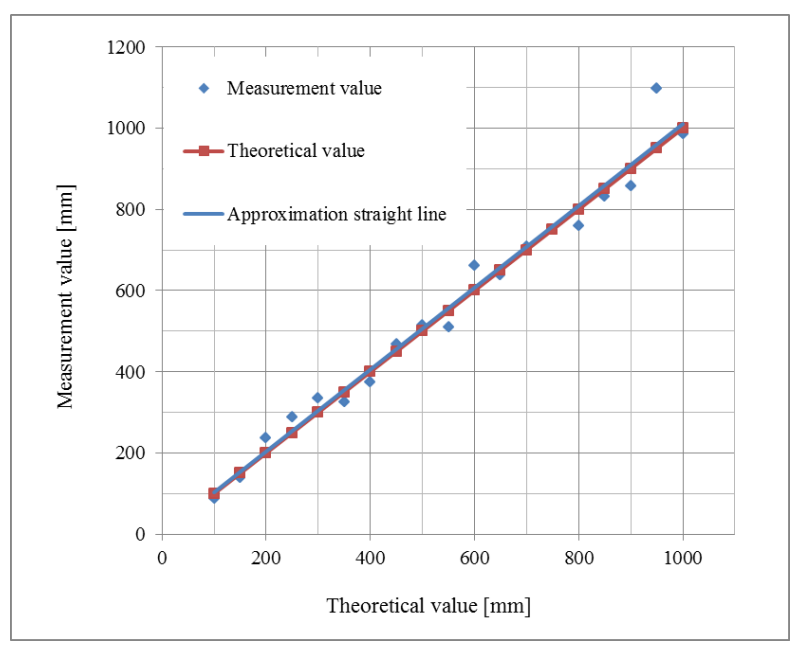

(a) $\Delta X=10[\mathrm{~cm}]$

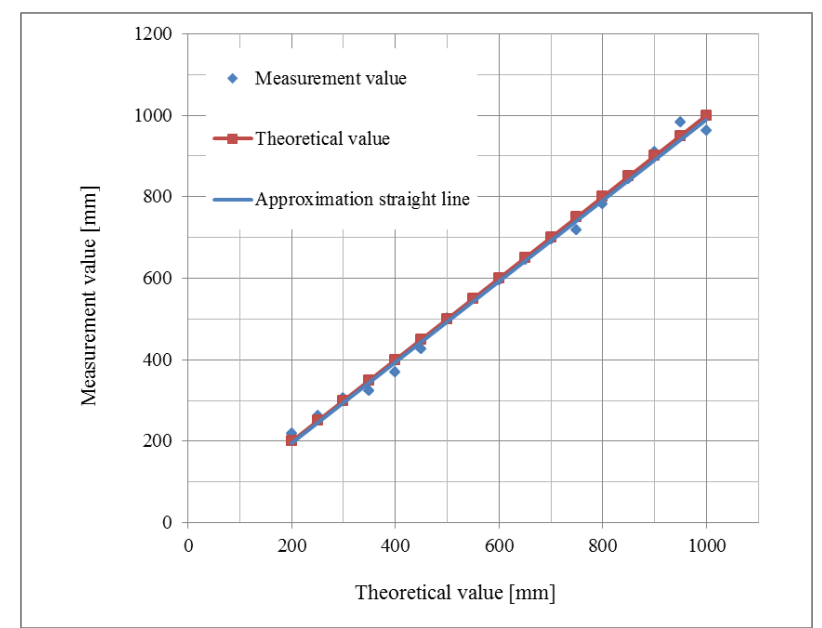

(b) $\triangle X=20[\mathrm{~cm}]$

Fig. 6. Accuracy comparison on the light source 
$Z$ coordinate was moved from $10[\mathrm{~cm}]$ to $100[\mathrm{~cm}]$ at an interval of $5[\mathrm{~cm}]$.

We show the $Z$ coordinate measurement result when the light source interval $\Delta X$ was $10[\mathrm{~cm}], 20[\mathrm{~cm}]$ in Fig. 6(a), (b).

We describe the measurement result in the following. The 3D coordinate could not be measured because the marker was not entered into the view angle of the camera when the $Z$ coordinate was $10[\mathrm{~cm}]$ and 15 [cm]. From Fig. 5 and Fig. 6, the measurement accuracy of the wide type was more improved than the normal type. The spot light interval $\Delta x$ on the photosensitive area was widened by widening the light source interval $\Delta X$. Therefore, the measurement accuracy was improved, because the influence of the spot light displacement or quantization error was suppressed. However, if the light source interval is set excessively wide, it is difficult to enter the spot light of the marker on the photosensitive area. As the result, the $3 \mathrm{D}$ measurement area is narrowed. Therefore, the light source interval $\Delta X$ must be set to an appropriate value corresponding to application or the purpose.

\subsection{Accuracy verification of the bright type}

This experiment was the bright type. This type was the experiment in the case of using the high luminance light sources. We compared the measurement accuracy of the bright type and the normal type. In this experiment, the light source interval $\Delta X$ was $5[\mathrm{~cm}]$. Also, the radiant intensity of IREDs was 40 [mW/Sr]

First, we measured the $Z$ coordinate of the marker. The $X$ coordinate of two light sources of a marker were -2.5 $[\mathrm{cm}]$ and $+2.5[\mathrm{~cm}]$, The $Y$ coordinate of the marker was 0 [cm]. Moreover, the $Z$ coordinate of the marker was moved from $10[\mathrm{~cm}]$ to $100[\mathrm{~cm}]$ at an interval of $5[\mathrm{~cm}]$. We show the $Z$ coordinate measurement result in Fig. 7 .

Second, we measured the $X$ coordinate of the marker. The $Y$ coordinate of the marker was $0[\mathrm{~cm}]$, The $Z$ coordinate of the marker was $20[\mathrm{~cm}]$. Moreover, the $X$ coordinate of the marker was moved from $-20[\mathrm{~cm}]$ to +20 [cm] at an interval of $5[\mathrm{~cm}]$. In the similar way, the $X$ coordinate was measured when the $Z$ coordinate was 50 $[\mathrm{cm}]$ and $80[\mathrm{~cm}]$. We show the $X$ coordinate measurement result when the $Z$ coordinate was $20[\mathrm{~cm}], 50[\mathrm{~cm}]$ and 80 [cm] in Fig. 8(a), (b), (c).

We describe the measurement result in the following. First, we describe the $Z$ coordinate measurement result. From Fig. 7, the maximum measurement error was 54.1 $[\mathrm{mm}]$ and the maximum measurement error rate was 6.8
[\%]. From Fig. 4 and Fig. 7, the measurement error was more improved $78.9[\mathrm{~mm}]$, the measurement error rate was more improved 10.2 percentage points than the normal type.

Second, we describe the $X$ coordinate measurement result when the $Z$ coordinate was $20[\mathrm{~cm}]$. The 3D coordinate could not be measured because the marker was not entered into the view angle of the camera when the $X$ coordinate was $15[\mathrm{~cm}]$ or more in absolute value. From Fig. 8(a), the maximum measurement error was 10.7 [mm] and the maximum measurement error rate was 10.7 [\%]. From, Fig. 5(a) and Fig. 8(a), the measurement error was more improved $3.0[\mathrm{~mm}]$, the measurement error rate more was improved 3.0 percentage points than the normal type.

Third, we describe the $X$ coordinate measurement result when the $Z$ coordinate was 50 [cm]. From Fig. 8(b), the maximum measurement error was 10.1 [mm] and the maximum measurement error rate was 9.5 [\%]. From, Fig. 5(b) and Fig. 8(b), the measurement error was more improved 53.2 [mm], the measurement error rate was more improved 22.2 percentage points than the normal type.

Finally, we describe the $X$ coordinate measurement result when the $Z$ coordinate was 80 [cm]. From Fig. 8(c), the maximum measurement error was $21.6[\mathrm{~mm}]$ and the maximum measurement error rate was 10.8 [\%]. From Fig. 5(c) and Fig. 8(c), the measurement error was more improved 24.3 [mm], the measurement error rate was more improved 12.2 percentage points than the normal type.

In every case, the measurement accuracy was more improved than the normal type. From the above, it was confirmed that the measurement accuracy was correlation with the incident light quantity. The position resolution of the 2D PSD is changed by the signal photocurrent value.

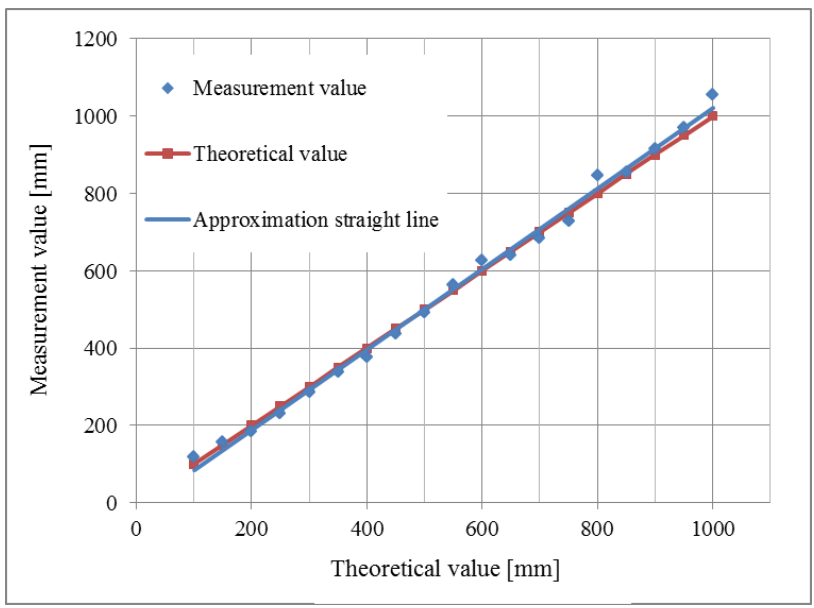

Fig. 7. Measurement result of the $Z$ coordinate when using the high luminance IREDs. 


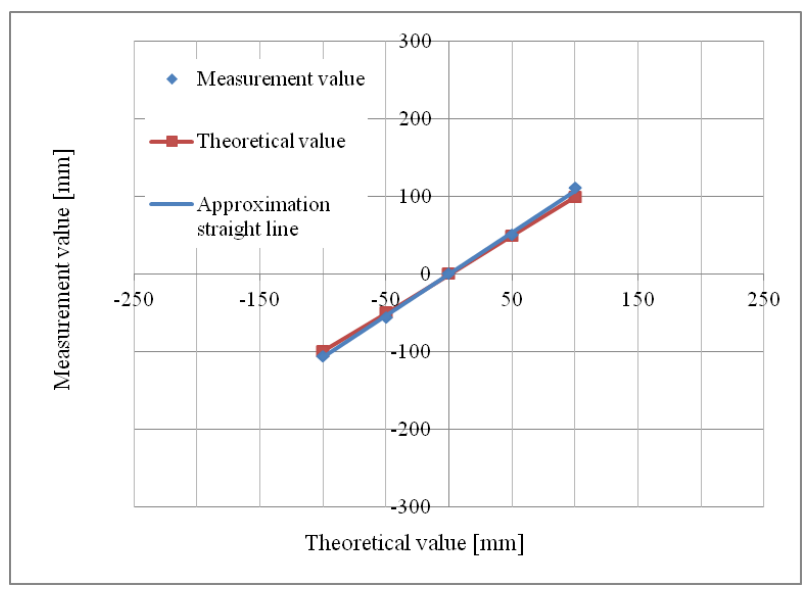

(a) $Z=20[\mathrm{~cm}]$

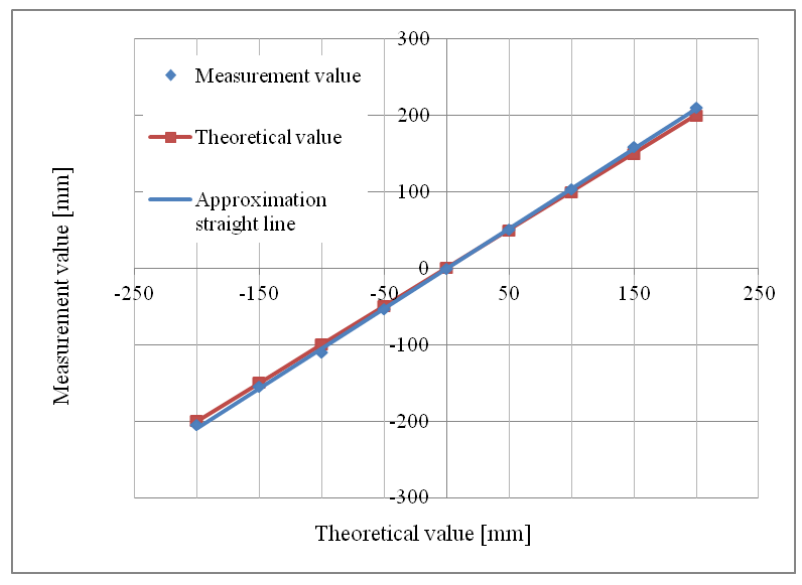

(b) $Z=50[\mathrm{~cm}]$

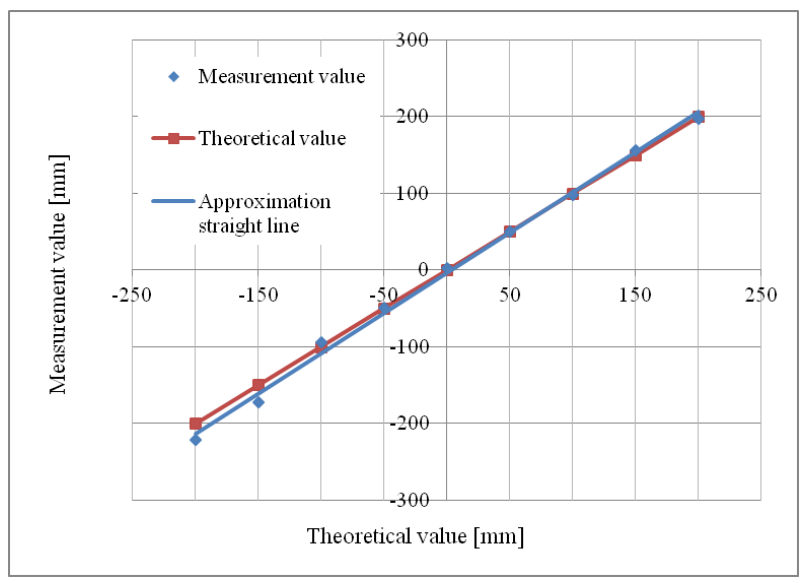

(c) $Z=80[\mathrm{~cm}]$

Fig. 8. Measurement result of the $X$ coordinate when using the high luminance IREDs.

Furthermore, the signal photocurrent value is in proportion to the incident light quantity. Therefore, the measurement accuracy is correlation with the incident light quantity.

\subsection{Accuracy verification of the zoom type}

From the above results of the wide type, one of the error factors was the narrow spot light interval on the photosensitive area. Because the bad influence of the temporal dispersion of spot light on measurement accuracy is large when the spot light interval was not larger than the error. However, the spot light interval is narrow when the distance between the 2D PSD and the marker is far as in Fig. 9. Therefore, we attempted improvement in measurement accuracy by controlling the photographing magnification.

This experiment was the zoom type. This type was the experiment in the case of using the high photographing magnification. We compared the measurement accuracy of the zoom type and the bright type with the smallest temporal dispersion of spot light. For the condition of this experiment, the light source interval $\Delta X$ was $5[\mathrm{~cm}]$, the radiant intensity of IREDs was $40[\mathrm{~mW} / \mathrm{Sr}]$ as with the condition of the bright type. The photographing magnification was 0.1 times when the $Z$ coordinate was 100 $[\mathrm{cm}]$ in the zoom type. Also, the photographing magnification was 0.7 times when the $Z$ coordinate was 200 [cm]. The spot light interval $2.4[\mathrm{~mm}]$ when the $Z$ coordinate was $10[\mathrm{~cm}]$ in the bright type as the comparison type. Therefore, we controlled the photographing magnification so that the spot light interval was about 2.4 [mm]. The distance $d$ is changed when the photographing magnification is changed. The distance $d$ was preset 45.1 [mm] when the photographing magnification was 0.1 times from experimental results. Also, the distance $d$ was preset $90.5[\mathrm{~mm}]$ when the photographing magnification was 0.7 times from experimental results. For the procedure, the measurement was performed five times in the same coordinate, compared the temporal dispersion of the average value.

We describe the measurement result in the following. First, we describe the accuracy comparison in the same spot light interval and the different $Z$ coordinate. We show the temporal dispersion of the measured $Z$ coordinate in Fig. 10. The temporal dispersion of the $Z$ coordinate was larger than other results when the $Z$ coordinate was $200[\mathrm{~cm}]$ in the zoom type because the incident light quantity was not enough. However, although we could not perform measurement when the $Z$ coordinate was $200[\mathrm{~cm}]$ in the bright type, we could perform highly precise measurement in the zoom type. Moreover, the temporal dispersion of the measured $Z$ coordinate was $0.73[\mathrm{~mm}]$ when the $Z$ coordinate was $100[\mathrm{~cm}]$ in the zoom type.

Second, we describe the accuracy comparison of each 
type in the same $Z$ coordinate. We show the temporal dispersion of the measured $Z$ coordinate in the same $Z$ coordinate in Fig. 11. From Fig. 11, the temporal dispersion of the $Z$ coordinate was more improved 59.2 [mm] than the bright type.

The experimental result shows that the zoom type is an effectual method to measure long distance target.

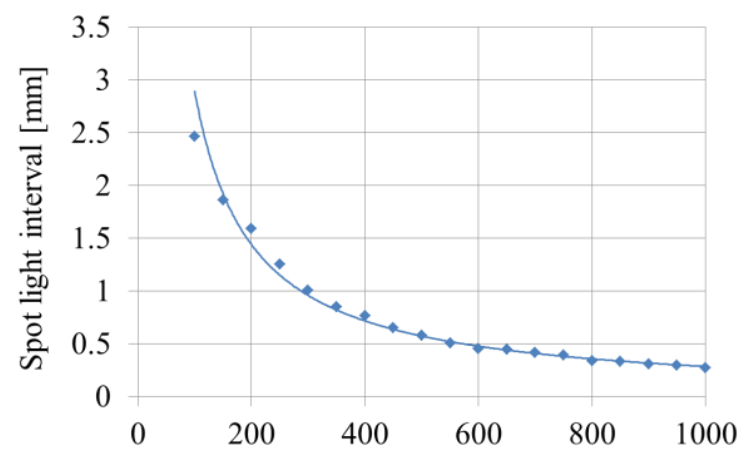

Distance between the 2D PSD and the marker [mm]

Fig. 9. The spot light interval to change of the distance between the 2D PSD and the marker.

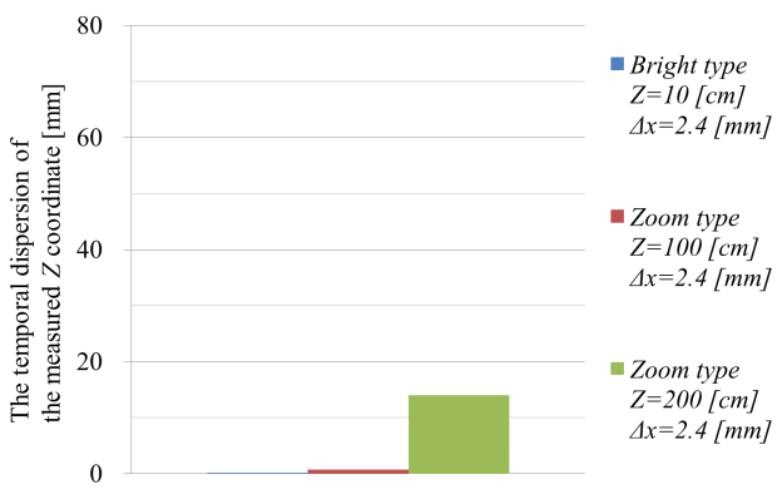

Fig. 10. The temporal dispersion of the measured $Z$ coordinate.

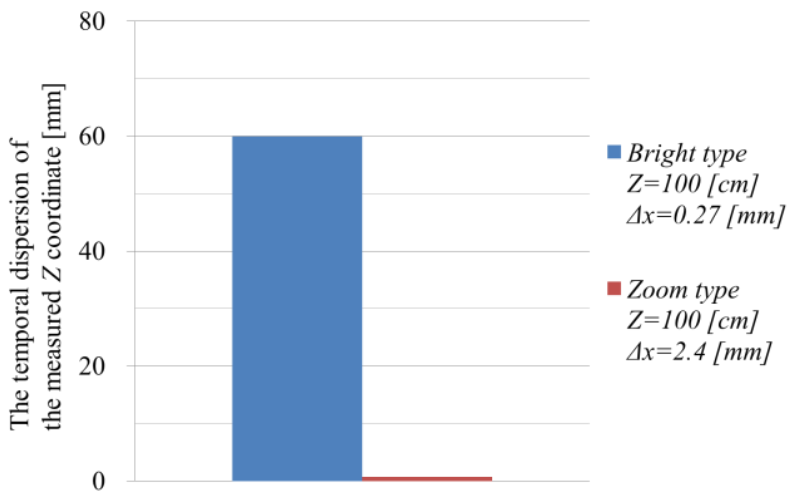

Fig. 11. The temporal dispersion of the measured $Z$ coordinate in the same $Z$ coordinate.

\section{Conclusions}

In this study, we proposed the 3D measurement method based on the new principle. The proposed method is able to detect the 3D position by a 2D PSD. Therefore, the proposed method is achieved at simpler preset and higher speed than other methods. Also, the proposed method was achieved at lower price than the multi camera stereo method. Moreover, the proposed method needs not reflect the light on the object surface because of using the marker. As a result, the proposed method is not influenced by the reflectance of the object surface. However, this proposed method has a condition that the marker must be arranged in parallel to the photosensitive area. Therefore, our future work is to solve this problem. The possible solution is to increase the number of light sources of a marker. Also, our future works are to improve the measurement accuracy and enlarge the measurement area.

\section{References}

(1) Yang, Qingxiong, et al. : "Fusion of active and passive sensors for fast $3 \mathrm{~d}$ capture" Multimedia Signal Processing (MMSP), 2010 IEEE International Workshop on. IEEE, pp. 69-74, 2010

(2) Yuya Kawahara, and Ryuichi Udo, and Lifeng Zhang : "High speed 3D Light Source Position Detection by Combining a 2D PSD an Ultrasonic Sensor Unit", Journal of the Institute of Industrial Applications Engineers, Vol. 2, No. 1, pp. 42-47, 2014

(3) Hua Li, and Jian Zhang, and Da Xu, and Mengchun Zhong: "Study on Laser Spot Affecting the Accuracy of Transient Angle Test System Based on PSD”, 3rd International Conference on Electric and Electronics (EEIC 2013), pp. 477-479, 2013

(4) Jeong-Gi Lee, and Do-Myung Kim, and Jin-Young Suk : "Development of a 3D Position Determination Device using PSD sensor", Control Automation and Systems (ICCAS), 2010 International Conference on, pp. 1733-1736, 2010

(5) Ying-gang Zhou, and Yan-gen Xie, and Dan Zhan, and Guang-ping Yu : "Research of 3D Spatial Localizing System Based on PSD Sensor", Measuring Technology and Mechatronics Automation, 2009. ICMTMA '09. International Conference on, Vol. 1, pp. 55-58, 2009 